\title{
On $\phi$-Ricci Recurrent Almost Kenmotsu Manifolds with Nullity Distributions
}

\author{
U.C. De* and Krishanu Mandal \\ (Communicated by Cihan Özgür)
}

\begin{abstract}
The purpose of this paper is to investigate $\phi$-Ricci recurrent and $\phi$-Ricci symmetric almost Kenmotsu manifolds with its characteristic vector field $\xi$ belonging to some nullity distributions. Also we obtain several corollaries. Finally, we give an example of a 5-dimensional almost Kenmotsu manifold such that $\xi$ belongs to the $(k, \mu)^{\prime}$-nullity distribution and $h^{\prime} \neq 0$.
\end{abstract}

Keywords: Almost Kenmotsu manifold; $\phi$-Ricci recurrence; $\phi$-Ricci symmetry; generalized nullity distribution; nullity distribution.

AMS Subject Classification (2010): Primary: 53C25 ; Secondary: 53D15.

\section{Introduction}

The notion of $k$-nullity distribution $(k \in \mathbb{R})$ was introduced by Gray [12] and Tanno [16] in the study of Riemannian manifolds $(M, g)$, which is defined for any $p \in M$ and $k \in \mathbb{R}$ as follows:

$$
N_{p}(k)=\left\{Z \in T_{p} M: R(X, Y) Z=k[g(Y, Z) X-g(X, Z) Y]\right\},
$$

for any $X, Y \in T_{p} M$, where $T_{p} M$ denotes the tangent vector space of $M$ at any point $p \in M$ and $R$ denotes the Riemannian curvature tensor of type $(1,3)$.

Recently, Blair, Koufogiorgos and Papantoniou [3] introduced the $(k, \mu)$-nullity distribution which is a generalized notion of the $k$-nullity distribution on a contact metric manifold $\left(M^{2 n+1}, \phi, \xi, \eta, g\right)$ and defined for any $p \in M^{2 n+1}$ and $k, \mu \in \mathbb{R}$ as follows:

$$
\begin{aligned}
N_{p}(k, \mu)=\left\{Z \in T_{p} M^{2 n+1}: R(X, Y) Z=\right. & k[g(Y, Z) X-g(X, Z) Y] \\
& +\mu[g(Y, Z) h X-g(X, Z) h Y]\},
\end{aligned}
$$

where $h=\frac{1}{2} £_{\xi} \phi$ and $£$ denotes the Lie differentiation.

Next, Dileo and Pastore [10] introduced another generalized notion of the $k$-nullity distribution which is named the $(k, \mu)^{\prime}$-nullity distribution on an almost Kenmotsu manifold $\left(M^{2 n+1}, \phi, \xi, \eta, g\right)$ and is defined for any $p \in M^{2 n+1}$ and $k, \mu \in \mathbb{R}$ as follows:

$$
\begin{aligned}
N_{p}(k, \mu)^{\prime}=\left\{Z \in T_{p} M^{2 n+1}: R(X, Y) Z=\right. & k[g(Y, Z) X-g(X, Z) Y] \\
& \left.+\mu\left[g(Y, Z) h^{\prime} X-g(X, Z) h^{\prime} Y\right]\right\}
\end{aligned}
$$

where $h^{\prime}=h \circ \phi$.

In [9], Dileo and Pastore studied locally symmetric almost Kenmotsu manifolds. We refer the reader to ([9],[10],[11]) for more related results on $(k, \mu)^{\prime}$-nullity distribution and $(k, \mu)$-nullity distribution on almost Kenmotsu manifolds. In recent papers ([17],[18],[19],[20],[21]) Wang and Liu study almost Kenmotsu manifolds with nullity distributions. In [19], Wang and Liu studied $\phi$-recurrent almost Kenmotsu manifolds with the characteristic vector field $\xi$ belonging to some nullity distributions.

On the other hand, Kenmotsu [13] introduced a special class of almost contact metric manifolds named Kenmotsu manifolds nowadays. The notion of locally $\phi$-symmetry was introduced by Takahashi [15] in the study of Sasakian manifolds as a weaker version of local symmetric of such manifolds. De et al [6] introduced 
a generalized version of local $\phi$-symmetry, called $\phi$-recurrence on Sasakian manifolds. In [5], De and Sarkar studied $\phi$-Ricci symmetric Sasakian manifolds. For more related results we refer the reader to De [4] and De et al ([7],[8]). Motivated by the above studies in this paper we investigate $\phi$-Ricci recurrent and $\phi$-Ricci symmetric almost Kenmotsu manifolds.

The paper is organized as follows:

In section 2, we give some basic knowledge on almost Kenmotsu manifolds. A few well-known results on almost Kenmotsu manifolds with $\xi$ belonging to some nullity distributions are provided in section 3 . In the next section, we study $\phi$-Ricci recurrent almost Kenmotsu manifolds with $\xi$ belonging to some nullity distributions. Also we obtain several corollaries. In the final section, we give an example of a 5-dimensional almost Kenmotsu manifold such that $\xi$ belongs to the $(k, \mu)^{\prime}$-nullity distribution and $h^{\prime} \neq 0$.

\section{Almost Kenmotsu manifolds}

Let $M$ be a $(2 n+1)$-dimensional differentiable manifold admits a $(\phi, \xi, \eta)$-structure or an almost contact structure, where $\phi$ is an $(1,1)$ tensor field, $\xi$ a characteristic vector field and $\eta$ an 1 -form such that $([1,2])$

$$
\phi^{2}=-I+\eta \otimes \xi, \eta(\xi)=1
$$

where $I$ denote the identity endomorphism. It is customary to include also $\phi \xi=0$ and $\eta \circ \phi=0$; both can be derived from (2.1).

If a manifold $M$ with a $(\phi, \xi, \eta)$-structure admits a Riemannian metric $g$ such that

$$
g(\phi X, \phi Y)=g(X, Y)-\eta(X) \eta(Y),
$$

for any vector fields $X$ and $Y$ of $T_{p} M^{2 n+1}$, then $M$ is said to have an almost contact metric structure $(\phi, \xi, \eta, g)$. The fundamental 2 -form $\Phi$ on an almost contact metric manifold is defined by $\Phi(X, Y)=g(X, \phi Y)$ for any vector fields $X, Y$ of $T_{p} M^{2 n+1}$. An almost Kenmotsu manifold is defined as an almost contact metric manifold such that $d \eta=0$ and $d \Phi=2 \eta \wedge \Phi$. The condition for an almost contact metric manifold being normal is equivalent to vanishing of the $(1,2)$-type torsion tensor $N_{\phi}$, defined by $N_{\phi}=[\phi, \phi]+2 d \eta \otimes \xi$, where $[\phi, \phi]$ is the Nijenhuis torsion of $\phi$ [1]. A normal almost Kenmotsu manifold is a Kenmotsu manifold. Also Kenmotsu manifolds can be characterized by $\left(\nabla_{X} \phi\right) Y=g(\phi X, Y) \xi-\eta(Y) \phi X$, for any vector fields $X, Y$. It is well known [13] that a Kenmotsu manifold $M^{2 n+1}$ is locally a warped product $I \times{ }_{f} N^{2 n}$ where $N^{2 n}$ is a Kähler manifold, $I$ is an open interval with coordinate $t$ and the warping function $f$, defined by $f=c e^{t}$ for some positive constant $c$. Let $\mathcal{D}$ be the distribution orthogonal to $\xi$ and defined by $\mathcal{D}=\operatorname{Ker}(\eta)=\operatorname{Im}(\phi)$. In an almost Kenmotsu manifold $\mathcal{D}$ is an integrable distribution as $\eta$ is closed. Let the two tensor fields $h=\frac{1}{2} £_{\xi} \phi$ and $l=R(\cdot, \xi) \xi$ on an almost Kenmotsu manifold $M^{2 n+1}$. The tensor fields $l$ and $h$ are symmetric and satisfy the following relations [14]

$$
\begin{gathered}
h \xi=0, l \xi=0, \operatorname{tr}(h)=0, \operatorname{tr}(h \phi)=0, h \phi+\phi h=0, \\
\nabla_{X} \xi=-\phi^{2} X-\phi h X\left(\Rightarrow \nabla_{\xi} \xi=0\right), \\
\phi l \phi-l=2\left(h^{2}-\phi^{2}\right), \\
R(X, Y) \xi=\eta(X)(Y-\phi h Y)-\eta(Y)(X-\phi h X)+\left(\nabla_{Y} \phi h\right) X-\left(\nabla_{X} \phi h\right) Y,
\end{gathered}
$$

for any vector fields $X, Y$. On the other hand, according to Takahashi [15] and De and Sarkar [5] we have the following definitions.

Definition 2.1. An almost Kenmotsu manifold is said to be $\phi$-symmetric, if it satisfies

$$
\phi^{2}\left(\left(\nabla_{W} R\right)(X, Y) Z\right)=0,
$$

for any vector fields $W, X, Y, Z \in T_{p} M$. In addition, if the vector fields $W, X, Y, Z$ are orthogonal to $\xi$, then the manifold is called locally $\phi$-symmetric manifold.

Definition 2.2. An almost Kenmotsu manifold is said to be $\phi$-Ricci recurrent if it satisfies

$$
\phi^{2}\left(\left(\nabla_{W} Q\right) Y\right)=A(W) Q Y
$$

for any vector fields $W, Y \in T_{p} M$, where $A$ is the 1 -form on $M^{2 n+1}$ and $Q$ is the Ricci operator defined by $S(X, Y)=g(Q X, Y)$. In addition, if the vector fields $W, Y$ are orthogonal to $\xi$, then the manifold is called locally $\phi$-Ricci recurrent manifold. 
Definition 2.3. An almost Kenmotsu manifold is said to be $\phi$-Ricci symmetric if it satisfies

$$
\phi^{2}\left(\left(\nabla_{W} Q\right) Y\right)=0,
$$

for any vector fields $W, Y \in T_{p} M$, where $Q$ is the Ricci operator defined by $S(X, Y)=g(Q X, Y)$. In addition, if the vector fields $W, Y$ are orthogonal to $\xi$, then the manifold is called locally $\phi$-Ricci symmetric manifold.

\section{Properties of the nullity conditions}

In this section we provide some related results on almost Kenmotsu manifolds with $\xi$ belonging to some nullity distributions. The (1,1)-type symmetric tensor field $h^{\prime}=h \circ \phi$ is anticommuting with $\phi$ and $h^{\prime} \xi=0$. Also it is clear that

$$
h=0 \Leftrightarrow h^{\prime}=0, \quad h^{\prime 2}=(k+1) \phi^{2}\left(\Leftrightarrow h^{2}=(k+1) \phi^{2}\right) .
$$

Let $X \in \mathcal{D}$ be the eigen vector of $h^{\prime}$ corresponding to the eigen value $\lambda$. It follows from (3.1) that $\lambda^{2}=-(k+1)$, a constant. Therefore $k \leq-1$ and $\lambda= \pm \sqrt{-k-1}$. We denote by $[\lambda]^{\prime}$ and $[-\lambda]^{\prime}$ the corresponding eigenspaces associated with $h^{\prime}$ corresponding to the non-zero eigen value $\lambda$ and $-\lambda$ respectively. We have following lemmas.

Lemma 3.1. (Proposition 3.1 and Proposition 5.1 of [14]) Let $\left(M^{2 n+1}, \phi, \xi, \eta, g\right)$ be an almost Kenmotsu manifold satisfying either the generalized $(k, \mu)$-nullity condition or the generalized $(k, \mu)^{\prime}$-nullity condition (the term generalized means $k, \mu$ both are smooth functions ), with $h \neq 0$. Then, one has

$$
\begin{gathered}
h^{\prime 2}=(k+1) \phi^{2}\left(\Leftrightarrow h^{2}=(k+1) \phi^{2}\right), \\
S(X, \xi)=2 n k \eta(X),
\end{gathered}
$$

for any vector field $X$ on $M^{2 n+1}$. Furthermore, in the case of generalized $(k, \mu)$-nullity condition, one has

$$
R(\xi, X) Y=k[g(X, Y) \xi-\eta(Y) X]+\mu[g(h X, Y) \xi-\eta(Y) h X]
$$

and in the case of generalized $(k, \mu)^{\prime}$-nullity condition, one has

$$
R(\xi, X) Y=k[g(X, Y) \xi-\eta(Y) X]+\mu\left[g\left(h^{\prime} X, Y\right) \xi-\eta(Y) h^{\prime} X\right],
$$

for any $X, Y \in T_{p} M$. In addition if $n>1$ then one has

$$
\left(\nabla_{X} h^{\prime}\right) Y=-g\left(h^{\prime} X+h^{\prime 2} X, Y\right) \xi-\eta(Y)\left(h^{\prime} X+h^{\prime 2} X\right)-(\mu+2) \eta(X) h^{\prime} Y,
$$

for any $X, Y \in T_{p} M$.

Lemma 3.2. (Proposition 4.1 and Proposition 4.3 of [10]) Let $\left(M^{2 n+1}, \phi, \xi, \eta, g\right)$ be an almost Kenmotsu manifold such that $\xi$ belongs to the $(k, \mu)^{\prime}$-nullity distribution and $h^{\prime} \neq 0$. Then $k<-1, \mu=-2$ and Spec $\left(h^{\prime}\right)=\{0, \lambda,-\lambda\}$, with 0 as simple eigen value and $\lambda=\sqrt{-k-1}$. The distributions $[\xi] \oplus[\lambda]^{\prime}$ and $[\xi] \oplus[-\lambda]^{\prime}$ are integrable with totally geodesic leaves. The distributions $[\lambda]^{\prime}$ and $[-\lambda]^{\prime}$ are integrable with totally umbilical leaves. Furthermore, the sectional curvature are given as following:

(a) $K(X, \xi)=k-2 \lambda$ if $X \in[\lambda]^{\prime}$ and $K(X, \xi)=k+2 \lambda$ if $X \in[-\lambda]^{\prime}$

(b) $K(X, Y)=k-2 \lambda$ if $X, Y \in[\lambda]^{\prime}$; $K(X, Y)=k+2 \lambda$ if $X, Y \in[-\lambda]^{\prime}$ and $K(X, Y)=-(k+2)$ if $X \in[\lambda]^{\prime}, Y \in[-\lambda]^{\prime}$,

(c) $M^{2 n+1}$ has constant negative scalar curvature $r=2 n(k-2 n)$.

Lemma 3.3. (Lemma 3 of [19]) Let $\left(M^{2 n+1}, \phi, \xi, \eta, g\right)$ be an almost Kenmotsu manifold with $\xi$ belonging to the generalized $(k, \mu)^{\prime}$-nullity distribution and $h^{\prime} \neq 0$. If $n>1$, then the Ricci operator $Q$ of $M^{2 n+1}$ is given by

$$
Q=-2 n i d+2 n(k+1) \eta \otimes \xi+[\mu-2(n-1)] h^{\prime} .
$$

Moreover, if both $k$ and $\mu$ are constant, then we have

$$
Q=-2 n i d+2 n(k+1) \eta \otimes \xi-2 n h^{\prime} .
$$

In both cases, the scalar curvature of $M^{2 n+1}$ is $2 n(k-2 n)$. 
Lemma 3.4. (Proposition 4.2 of [10]) Let $\left(M^{2 n+1}, \phi, \xi, \eta, g\right)$ be an almost Kenmotsu manifold such that $h^{\prime} \neq 0$ and $\xi$ belongs to the $(k,-2)^{\prime}$-nullity distribution. Then for any $X_{\lambda}, Y_{\lambda}, Z_{\lambda} \in[\lambda]^{\prime}$ and $X_{-\lambda}, Y_{-\lambda}, Z_{-\lambda} \in[-\lambda]^{\prime}$, the Riemannian curvature tensor satisfies:

$$
\begin{aligned}
R\left(X_{\lambda}, Y_{\lambda}\right) Z_{-\lambda} & =0 \\
R\left(X_{-\lambda}, Y_{-\lambda}\right) Z_{\lambda} & =0 \\
R\left(X_{\lambda}, Y_{-\lambda}\right) Z_{\lambda} & =(k+2) g\left(X_{\lambda}, Z_{\lambda}\right) Y_{-\lambda} \\
R\left(X_{\lambda}, Y_{-\lambda}\right) Z_{-\lambda} & =-(k+2) g\left(Y_{-\lambda}, Z_{-\lambda}\right) X_{\lambda} \\
R\left(X_{\lambda}, Y_{\lambda}\right) Z_{\lambda} & =(k-2 \lambda)\left[g\left(Y_{\lambda}, Z_{\lambda}\right) X_{\lambda}-g\left(X_{\lambda}, Z_{\lambda}\right) Y_{\lambda}\right] \\
R\left(X_{-\lambda}, Y_{-\lambda}\right) Z_{-\lambda} & =(k+2 \lambda)\left[g\left(Y_{-\lambda}, Z_{-\lambda}\right) X_{-\lambda}-g\left(X_{-\lambda}, Z_{-\lambda}\right) Y_{-\lambda}\right] .
\end{aligned}
$$

Lemma 3.5. (Lemma 4.1 of [10]) Let $\left(M^{2 n+1}, \phi, \xi, \eta, g\right)$ be an almost Kenmotsu manifold with $h^{\prime} \neq 0$ and $\xi$ belonging to the $(k,-2)^{\prime}$-nullity distribution. Then for any $X, Y \in T_{p} M$,

$$
\left(\nabla_{X} h^{\prime}\right) Y=-g\left(h^{\prime} X+h^{\prime 2} X, Y\right) \xi-\eta(Y)\left(h^{\prime} X+h^{\prime 2} X\right) .
$$

Lemma 3.6. (Theorem 4.1 of [10]) Let $M$ be an almost Kenmotsu manifold of dimension $2 n+1$. Suppose that the characteristic vector field $\xi$ belonging to the $(k, \mu)$-nullity distribution. Then $k=-1, h=0$ and $M$ is locally a warped product of an open interval and an almost Kähler manifold.

\section{4. $\phi$-Ricci recurrent almost Kenmotsu manifolds}

This section is devoted to study $\phi$-Ricci recurrent almost Kenmotsu manifolds with some nullity distributions. At first we prove the following theorem.

Theorem 4.1. Let $\left(M^{2 n+1}, \phi, \xi, \eta, g\right)(n>1)$ be a $\phi$-Ricci recurrent almost Kenmotsu manifold with the characteristic vector field $\xi$ belonging to the $(k, \mu)^{\prime}$-nullity distribution and $h^{\prime} \neq 0$. Then the manifold $M^{2 n+1}$ is $\phi$-Ricci symmetric and hence locally isometric to the Riemannian product of an $(n+1)$-dimensional manifold of constant sectional curvature -4 and a flat $n$-dimensional manifold.

Proof. We suppose that the manifold $M^{2 n+1}$ is a $\phi$-Ricci recurrent almost Kenmotsu manifold. Taking the covariant differentiation along arbitrary vector field $Y \in T_{p} M$ of (3.8) we have

$$
\left(\nabla_{Y} Q\right) X=2 n(k+1)\left[\left(\nabla_{Y} \eta\right) X \xi+\eta(X) \nabla_{Y} \xi\right]-2 n\left(\nabla_{Y} h^{\prime}\right) X
$$

for any vector fields $X, Y \in T_{p} M$. Using (2.3) we obtain from the above equation

$$
\begin{aligned}
\left(\nabla_{Y} Q\right) X= & 2 n(k+1)[g(X, Y) \xi-2 \eta(X) \eta(Y) \xi+Y \eta(X) \\
& \left.+g\left(X, h^{\prime} Y\right) \xi+h^{\prime} Y \eta(X)\right]-2 n\left(\nabla_{Y} h^{\prime}\right) X,
\end{aligned}
$$

for any vector fields $X, Y \in T_{p} M$. Applying $\phi^{2}$ on both sides of (4.2) we obtain

$$
\begin{aligned}
\phi^{2}\left(\left(\nabla_{Y} Q\right) X\right)= & 2 n(k+1)\left[-\eta(X) Y+\eta(X) \eta(Y) \xi-\eta(X) h^{\prime} Y\right] \\
& -2 n \phi^{2}\left(\left(\nabla_{Y} h^{\prime}\right) X\right),
\end{aligned}
$$

for any vector fields $X, Y \in T_{p} M$. Making use of (3.9) we get from (4.3)

$$
\begin{aligned}
\phi^{2}\left(\left(\nabla_{Y} Q\right) X\right)= & 2 n(k+1)\left[-\eta(X) Y+\eta(X) \eta(Y) \xi-\eta(X) h^{\prime} Y\right] \\
& +2 n \eta(X)\left[-h^{\prime} Y+(k+1)(Y-\eta(Y) \xi)\right],
\end{aligned}
$$

from which we have

$$
\phi^{2}\left(\left(\nabla_{Y} Q\right) X\right)=-2 n(k+2) \eta(X) h^{\prime} Y
$$

for any vector fields $X, Y \in T_{p} M$. By virtue of equations (4.5) and (2.6) we obtain

$$
-2 n(k+2) \eta(X) h^{\prime} Y=A(Y) Q X
$$

for any vector fields $X, Y \in T_{p} M$. Substituting $X=\xi$ in (4.6) yields

$$
-(k+2) h^{\prime} Y=k A(Y) \xi,
$$


for any vector field $Y \in T_{p} M$. Taking inner product of (4.7) with $\xi$ we have

$$
k A(Y)=0,
$$

for any vector field $Y \in T_{p} M$. Dileo and Pastore [10] proved that in an almost Kenmotsu manifold with the characteristic vector field $\xi$ belonging to the $(k, \mu)^{\prime}$-nullity distribution with $h^{\prime} \neq 0$ then $k<-1$. Hence (4.8) implies $A=0$, that is, the manifold $M^{2 n+1}$ is $\phi$-Ricci symmetric. Using the relation $A=0$, it follows from (4.7) that $k=-2$. Noticing the fact $\lambda^{2}=-(k+1)$ and $k=-2$ we have $\lambda= \pm 1$. Without losing generality we may choose $\lambda=1$. Then we can write from Lemma 3.4

$$
\begin{aligned}
R\left(X_{\lambda}, Y_{\lambda}\right) Z_{\lambda} & =-4\left[g\left(Y_{\lambda}, Z_{\lambda}\right) X_{\lambda}-g\left(X_{\lambda}, Z_{\lambda}\right) Y_{\lambda}\right] \\
R\left(X_{-\lambda}, Y_{-\lambda}\right) Z_{-\lambda} & =0
\end{aligned}
$$

for any $X_{\lambda}, Y_{\lambda}, Z_{\lambda} \in[\lambda]^{\prime}$ and $X_{-\lambda}, Y_{-\lambda}, Z_{-\lambda} \in[-\lambda]^{\prime}$. Also it follows from Lemma 3.2 that $K(X, \xi)=-4$ for any $X \in[\lambda]^{\prime}$ and $K(X, \xi)=0$ for any $X \in[-\lambda]^{\prime}$. Again from Lemma 3.2 we see that $K(X, Y)=-4$ for any $X, Y \in[\lambda]^{\prime} ; K(X, Y)=0$ for any $X, Y \in[-\lambda]^{\prime}$ and $K(X, Y)=0$ for any $X \in[\lambda]^{\prime}, Y \in[-\lambda]^{\prime}$. As is shown in [10] that the distribution $[\xi] \oplus[\lambda]^{\prime}$ is integrable with totally geodesic leaves and the distribution $[-\lambda]^{\prime}$ is integrable with totally umbilical leaves by $H=-(1-\lambda) \xi$, where $H$ is the mean curvature vector field for the leaves of $[-\lambda]^{\prime}$ immersed in $M^{2 n+1}$. Here $\lambda=1$, then two orthogonal distributions $[\xi] \oplus[\lambda]^{\prime}$ and $[-\lambda]^{\prime}$ are both integrable with totally geodesic leaves immersed in $M^{2 n+1}$. Then we can say that $M^{2 n+1}$ is locally isometric to $\mathbb{H}^{n+1}(-4) \times \mathbb{R}^{n}$. This completes the proof.

Since $\phi$-recurrent implies $\phi$-Ricci recurrent, we have the following:

Corollary 4.1. Let $\left(M^{2 n+1}, \phi, \xi, \eta, g\right)(n>1)$ be a $\phi$-recurrent almost Kenmotsu manifold with the characteristic vector field $\xi$ belonging to the $(k, \mu)^{\prime}$-nullity distribution and $h^{\prime} \neq 0$. Then the manifold is locally isometric to the Riemannian product of an $(n+1)$-dimensional manifold of constant sectional curvature -4 and a flat $n$-dimensional manifold.

The above corollary have been proved by Wang and Liu [19].

Theorem 4.2. Let $\left(M^{2 n+1}, \phi, \xi, \eta, g\right)(n>1)$ be a $\phi$-Ricci recurrent almost Kenmotsu manifold with the characteristic vector field $\xi$ belonging to the generalized $(k, \mu)^{\prime}$-nullity distribution and $h^{\prime} \neq 0$. Then the following statements are equivalent:

(i) $M^{2 n+1}$ is $\phi$-Ricci symmetric;

(ii) $k$ is a constant;

(iii) $\xi$ belongs to the $(k, \mu)^{\prime}$-nullity distribution.

Proof. Let us suppose $M^{2 n+1}$ be a $\phi$-Ricci recurrent almost Kenmotsu manifold with $\xi$ belonging to the generalized $(k, \mu)^{\prime}$-nullity distribution and $h^{\prime} \neq 0$. Taking the covariant differentiation along arbitrary vector field $W \in T_{p} M$ of (3.7) we get

$$
\begin{aligned}
\left(\nabla_{W} Q\right) Y= & 2 n W(k) \eta(Y) \xi+2 n(k+1)\left[\left(\nabla_{W} \eta\right) Y \xi+\eta(Y) \nabla_{W} \xi\right] \\
& +W(\mu) h^{\prime} Y+[\mu-2(n-1)]\left(\nabla_{W} h^{\prime}\right) Y,
\end{aligned}
$$

for any vector fields $W, Y \in T_{p} M$. Applying $\phi^{2}$ on both sides of (4.9), we have

$$
\begin{aligned}
\phi^{2}\left(\left(\nabla_{W} Q\right) Y\right)= & -W(\mu) h^{\prime} Y+[\mu-2(n-1)] \phi^{2}\left(\left(\nabla_{W} h^{\prime}\right) Y\right) \\
& +2 n(k+1) \eta(Y)\left\{-W+\eta(W) \xi-h^{\prime} W\right\},
\end{aligned}
$$

for any vector fields $W, Y \in T_{p} M$. In view of (4.10) and (2.6) we have

$$
\begin{aligned}
& -W(\mu) h^{\prime} Y+[\mu-2(n-1)] \phi^{2}\left(\left(\nabla_{W} h^{\prime}\right) Y\right) \\
& +2 n(k+1) \eta(Y)\left\{-W+\eta(W) \xi-h^{\prime} W\right\}=A(W) Q Y,
\end{aligned}
$$

for any vector fields $W, Y \in T_{p} M$. Making use of (3.6) and (4.11) we obtain

$$
\begin{aligned}
& -W(\mu) h^{\prime} Y+[\mu-2(n-1)]\left[-\eta(Y)\left\{-h^{\prime} W+(k+1)(W-\eta(W) \xi)\right\}\right. \\
& \left.+(\mu+2) \eta(W) h^{\prime} Y\right]-2 n(k+1)\left[\eta(Y) W-\eta(Y) \eta(W) \xi+\eta(Y) h^{\prime} W\right] \\
& =A(W) Q Y
\end{aligned}
$$


for any vector fields $W, Y \in T_{p} M$. Taking inner product with $\xi$ of (4.12) yields

$$
k A(W)=0,
$$

for any vector field $W \in T_{p} M$. From (3.2) we see that the smooth function $k$ satisfies $k<-1$. Hence it follows from (4.13) that $A=0$. Thus $M^{2 n+1}$ is $\phi$-Ricci symmetric. Therefore $(i) \Rightarrow(i i)$ is proved.

Taking into account $A=0$ we have from (4.12)

$$
\begin{aligned}
& -W(\mu) h^{\prime} Y+[\mu-2(n-1)]\left[-\eta(Y)\left\{-h^{\prime} W+(k+1)(W-\eta(W) \xi)\right\}\right. \\
& \left.+(\mu+2) \eta(W) h^{\prime} Y\right]-2 n(k+1)\left[\eta(Y) W-\eta(Y) \eta(W) \xi+\eta(Y) h^{\prime} W\right] \\
& =0
\end{aligned}
$$

for any vector fields $W, Y \in T_{p} M$. Substituting $Y=\xi$ in (4.14) we obtain

$$
[\mu-2(n-1)]\left[h^{\prime} W-(k+1)(W-\eta(W) \xi)\right]-2 n(k+1)\left[W-\eta(W) \xi+h^{\prime} W\right]=0,
$$

for any vector field $W \in T_{p} M$. Now taking inner product of (4.15) with the vector field $X$ we have

$$
\begin{aligned}
& {[\mu-2(n-1)]\left[g\left(h^{\prime} W, X\right)-(k+1)(g(W, X)-\eta(W) \eta(X))\right]} \\
& -2 n(k+1)\left[g(W, X)-\eta(W) \eta(X)+g\left(h^{\prime} W, X\right)\right]=0,
\end{aligned}
$$

for any vector fields $W, X \in T_{p} M$. Consider a local orthonormal basis $\left\{e_{i}: i=1,2, \ldots, 2 n+1\right\}$ of tangent space at each point of the manifold $M^{2 n+1}$. Setting $X=W=e_{i}$ in (4.16) and taking summation over $i: 1 \leq i \leq 2 n+1$, we get

$$
(k+1)(\mu+2)=0 .
$$

Again from (3.2) we see that the smooth function $k$ satisfies $k<-1$. Therefore it follows from (4.17) that $\mu=-2$. Pastore and Saltarelli [14] proved that in an almost Kenmotsu manifold with generalized $(k, \mu)^{\prime}$-nullity distribution and $h^{\prime} \neq 0$, then

$$
\xi(k)=-2(k+1)(\mu+2)
$$

holds. In view of $\mu=-2$ we have from (4.18), $k=$ constant. Thus $(i i) \Rightarrow(i i i)$ is proved. Conversely, $(i i i) \Rightarrow(i)$ is proved in Theorem 4.1. This completes the proof.

Since $\phi$-recurrent implies $\phi$-Ricci recurrent, we have the following:

Corollary 4.2. Let $\left(M^{2 n+1}, \phi, \xi, \eta, g\right)(n>1)$ be a $\phi$-recurrent almost Kenmotsu manifold with the characteristic vector field $\xi$ belonging to the generalized $(k, \mu)^{\prime}$-nullity distribution and $h^{\prime} \neq 0$. Then the following statements are equivalent:

(i) $M^{2 n+1}$ is $\phi$-symmetric;

(ii) $k$ is a constant;

(iii) $\xi$ belongs to the $(k, \mu)^{\prime}$-nullity distribution.

The above corollary have been proved by Wang and Liu [19].

Now we prove some special theorems on almost Kenmotsu manifolds with $\xi$ belonging to the $(k, \mu)$-nullity and generalized $(k, \mu)$-nullity distributions.

Theorem 4.3. Let $\left(M^{2 n+1}, \phi, \xi, \eta, g\right)$ be a $\phi$-Ricci recurrent almost Kenmotsu manifold with the characteristic vector field $\xi$ belonging to the $(k, \mu)$-nullity distribution. Then the manifold $M^{2 n+1}$ is an Einstein one.

Proof. Let us suppose that the manifold $M^{2 n+1}$ is an $\phi$-Ricci recurrent almost Kenmotsu manifold with $\xi$ belonging to the $(k, \mu)$-nullity distribution. In view of Lemma 3.6 and the equation (2.5) we get

$$
R(X, Y) \xi=\eta(X) Y-\eta(Y) X
$$

for any $X, Y \in T_{p} M$. Contracting $Y$ in (4.19) we have

$$
S(X, \xi)=-2 n \eta(X),
$$

for any $X, Y \in T_{p} M$. Thus, we have

$$
Q \xi=-2 n \xi
$$


Taking the covariant differentiation along arbitrary vector field $Z \in T_{p} M$ of (4.21) we get

$$
\left(\nabla_{Z} Q\right) \xi=-2 n\left(\nabla_{Z} \xi\right)
$$

Using Lemma 3.6 the Equation (2.3) becomes

$$
\nabla_{Z} \xi=Z-\eta(Z) \xi
$$

for any $Z \in T_{p} M$. From (4.22) and (4.23) we have

$$
\left(\nabla_{Z} Q\right) \xi=-2 n[Z-\eta(Z) \xi],
$$

where $Z$ be any vector field on $M$. Applying $\phi^{2}$ on both sides of (4.24) we see that

$$
\phi^{2}\left(\left(\nabla_{Z} Q\right) \xi\right)=-2 n[-Z+\eta(Z) \xi]
$$

for any $Z \in T_{p} M$. In view of (4.25) and (2.6) we obtain

$$
-2 n[-Z+\eta(Z) \xi]=A(Z) Q \xi,
$$

where $Z \in T_{p} M$. Taking the inner product of (4.26) with $\xi$ yields $A(Z)=0$, for any $Z \in T_{p} M$, thus we have $A=0$ and consequently $M^{2 n+1}$ is $\phi$-Ricci symmetric. Taking into account $A=0$, we can write from (4.25) and (4.26) that $\phi^{2}\left(\left(\nabla_{Z} Q\right) \xi\right)=0$, for any $Z \in T_{p} M$, from which it follows that

$$
-\left(\nabla_{Z} Q\right) \xi+\eta\left(\left(\nabla_{Z} Q\right) \xi\right) \xi=0 .
$$

Taking the inner product of (4.27) with the vector field $U$ and making use of $g\left(\left(\nabla_{Z} Q\right) \xi, U\right)=\left(\nabla_{Z} S\right)(\xi, U)$ we have

$$
\left(\nabla_{Z} S\right)(\xi, U)-\eta\left(\left(\nabla_{Z} Q\right) \xi\right) \eta(U)=0,
$$

for any $U, Z \in T_{p} M$. Again using (4.24) we see from (4.28) that

$$
\left(\nabla_{Z} S\right)(\xi, U)=0
$$

which implies

$$
\nabla_{Z} S(\xi, U)-S\left(\nabla_{Z} \xi, U\right)-S\left(\xi, \nabla_{Z} U\right)=0,
$$

for any $U, Z \in T_{p} M$. Making use of (4.20) and (4.23) into (4.30) we can write $S(U, Z)=-2 n g(U, Z)$, for any $U, Z \in T_{p} M$. Therefore the manifold $M^{2 n+1}$ is an Einstein manifold. This completes the proof.

In [19], Wang and Liu proved that a $\phi$-recurrent almost Kenmotsu manifold $M^{2 n+1}$ with the characteristic vector field $\xi$ belonging to the $(k, \mu)$-nullity distribution is of constant sectional curvature -1 , which implies that the manifold is an Einstein one of the form $S(U, Z)=-2 n g(U, Z)$, for any $U, Z \in T_{p} M$. Also, $\phi$-recurrent implies $\phi$-Ricci recurrent. Thus, we have the following:

Corollary 4.3. Let $\left(M^{2 n+1}, \phi, \xi, \eta, g\right)$ be a $\phi$-recurrent almost Kenmotsu manifold with the characteristic vector field $\xi$ belonging to the $(k, \mu)$-nullity distribution. Then the manifold $M^{2 n+1}$ is an Einstein one.

Theorem 4.4. Let $\left(M^{2 n+1}, \phi, \xi, \eta, g\right)$ be a $\phi$-Ricci recurrent almost Kenmotsu manifold with the characteristic vector field $\xi$ belonging to the generalized $(k, \mu)$-nullity distribution and $h \neq 0$. Then the manifold $M^{2 n+1}$ is an Einstein one.

Proof. Suppose that an almost Kenmotsu manifold $M^{2 n+1}$ is $\phi$-Ricci recurrent and $\xi$ belongs to the generalized $(k, \mu)$-nullity distribution with $h \neq 0$. Making use of (3.3) we get

$$
Q \xi=2 n k \xi .
$$

Taking the covariant differentiation along arbitrary vector field $Z \in T_{p} M$ of (4.31) we get

$$
\left(\nabla_{Z} Q\right) \xi=2 n k\left(\nabla_{Z} \xi\right)+2 n Z(k) \xi .
$$

Using (2.3) in (4.32) yields

$$
\left(\nabla_{Z} Q\right) \xi=2 n k(Z-\eta(Z) \xi-\phi h Z)+2 n Z(k) \xi
$$


for any vector field $Z \in T_{p} M$. Applying $\phi^{2}$ on both sides of (4.33) we have

$$
\phi^{2}\left(\left(\nabla_{Z} Q\right) \xi\right)=2 n k(-Z+\eta(Z) \xi+\phi h Z),
$$

for any vector field $Z \in T_{p} M$. On the other hand, the assumption that $M^{2 n+1}$ is $\phi$-Ricci recurrent implies $\phi^{2}\left(\left(\nabla_{Z} Q\right) Y\right)=A(Z) Q Y$, for any $Y, Z \in T_{p} M$. Substituting $Y=\xi$ into this relation we obtain

$$
\phi^{2}\left(\left(\nabla_{Z} Q\right) \xi\right)=A(Z) Q \xi
$$

In view of (4.34) and (4.35) we get

$$
2 n k(-Z+\eta(Z) \xi+\phi h Z)=A(Z) Q \xi,
$$

for any vector field $Z \in T_{p} M$. Taking inner product of (4.36) with $\xi$ implies $k A(Z)=0$ for any vector field $Z \in T_{p} M$. Also, from (3.2) we see that the smooth function $k$ satisfies the condition $k<-1$. Therefore, $A=0$ and consequently $M^{2 n+1}$ is $\phi$-Ricci symmetric. Taking into account $A=0$, we can write from (4.35) that $\phi^{2}\left(\left(\nabla_{Z} Q\right) \xi\right)=0$, for any $Z \in T_{p} M$, from which it follows that

$$
-\left(\nabla_{Z} Q\right) \xi+\eta\left(\left(\nabla_{Z} Q\right) \xi\right) \xi=0 .
$$

Taking the inner product of (4.37) with the vector field $U$ and using $g\left(\left(\nabla_{Z} Q\right) \xi, U\right)=\left(\nabla_{Z} S\right)(\xi, U)$ we have

$$
\left(\nabla_{Z} S\right)(\xi, U)-\eta\left(\left(\nabla_{Z} Q\right) \xi\right) \eta(U)=0
$$

for any vector fields $U, Z \in T_{p} M$. This implies

$$
\nabla_{Z} S(\xi, U)-S\left(\nabla_{Z} \xi, U\right)-S\left(\xi, \nabla_{Z} U\right)-\eta\left(\left(\nabla_{Z} Q\right) \xi\right) \eta(U)=0 .
$$

Applying (3.3), (2.3) and (4.33) into (4.39) we obtain

$$
\begin{aligned}
& 2 n Z(k) \eta(U)+2 n k \nabla_{Z} \eta(U)-S(Z, U)+2 n k \eta(Z) \eta(U) \\
& +S(\phi h Z, U)-2 n k \eta\left(\nabla_{Z} U\right)-2 n Z(k) \eta(U)=0,
\end{aligned}
$$

from which it follows that

$$
S(U, Z)-S(\phi h Z, U)-2 n k g(U, Z)+2 n k g(\phi h Z, U)=0,
$$

for any vector fields $U, Z \in T_{p} M$. Now replacing $Z$ by $\phi h Z$ in (4.41) and noticing the fact $h^{2}=(k+1) \phi^{2}$ we have

$$
(k+1) S(U, Z)+S(\phi h Z, U)-2 n k(k+1) g(U, Z)-2 n k g(\phi h Z, U)=0,
$$

for any vector fields $U, Z \in T_{p} M$. Adding (4.41) and (4.42) yields

$$
(k+2)[S(U, Z)-2 n k g(U, Z)]=0,
$$

for any vector fields $U, Z \in T_{p} M$. Clearly, it follows from (4.43) that either $k=-2$ or, $S(U, Z)=2 n k g(U, Z)$, for any vector fields $U, Z \in T_{p} M$. Now we prove that the former case can not occur. Indeed, if we assume that the former case is true, that is, $k=-2$, a constant, then $\xi(k)=0$. Here we recall a result due to Pastore and Saltarelli [14]. They proved that in an almost Kenmotsu manifold with generalized $(k, \mu)$-nullity distribution and $h \neq 0$, the relation $\xi(k)=-4(k+1)$ holds. Therefore substituting $k=-2$ in this relation we have $\xi(k)=4$. Thus, we have $\xi(k)=0$ and $\xi(k)=4$, which is absurd. Hence, we get the desired result.

\section{Example of a 5-dimensional almost Kenmotsu manifold}

In this section, we construct an example of an almost Kenmotsu manifold such that $\xi$ belongs to the $(k, \mu)^{\prime}-$ nullity distribution. We consider 5-dimensional manifold $M=\left\{(x, y, z, u, v) \in \mathbb{R}^{5}\right\}$, where $(x, y, z, u, v)$ are the standard coordinates in $\mathbb{R}^{5}$. Let $\xi, e_{2}, e_{3}, e_{4}, e_{5}$ are five vector fields in $\mathbb{R}^{5}$ which satisfies [10]

$$
\begin{aligned}
& {\left[\xi, e_{2}\right]=-2 e_{2},\left[\xi, e_{3}\right]=-2 e_{3},\left[\xi, e_{4}\right]=0,\left[\xi, e_{5}\right]=0,} \\
& {\left[e_{i}, e_{j}\right]=0, \text { where } i, j=2,3,4,5 .}
\end{aligned}
$$


Let $g$ be the Riemannian metric defined by

$$
\begin{aligned}
& g(\xi, \xi)=g\left(e_{2}, e_{2}\right)=g\left(e_{3}, e_{3}\right)=g\left(e_{4}, e_{4}\right)=g\left(e_{5}, e_{5}\right)=1 \\
& \text { and } g\left(\xi, e_{i}\right)=g\left(e_{i}, e_{j}\right)=0 \text { for } i \neq j ; i, j=2,3,4,5 .
\end{aligned}
$$

Let $\eta$ be the 1-form defined by

$$
\eta(Z)=g(Z, \xi),
$$

for any $Z \in \chi(M)$. Let $\phi$ be the $(1,1)$-tensor field defined by

$$
\phi(\xi)=0, \phi\left(e_{2}\right)=e_{4}, \phi\left(e_{3}\right)=e_{5}, \phi\left(e_{4}\right)=-e_{2}, \phi\left(e_{5}\right)=-e_{3} .
$$

Using the linearity of $\phi$ and $g$ we have

$$
\eta(\xi)=1, \phi^{2} Z=-Z+\eta(Z) \xi
$$

and

$$
g(\phi Z, \phi U)=g(Z, U)-\eta(Z) \eta(U)
$$

for any $U, Z \in \chi(M)$. Moreover,

$$
h^{\prime} \xi=0, h^{\prime} e_{2}=e_{2}, h^{\prime} e_{3}=e_{3}, h^{\prime} e_{4}=-e_{4}, h^{\prime} e_{5}=-e_{5} .
$$

The Levi-Civita connection $\nabla$ of the metric tensor $g$ is given by Koszul's formula which is given by

$$
\begin{aligned}
2 g\left(\nabla_{X} Y, Z\right)= & X g(Y, Z)+Y g(Z, X)-Z g(X, Y) \\
& -g(X,[Y, Z])-g(Y,[X, Z])+g(Z,[X, Y]) .
\end{aligned}
$$

Using Koszul's formula we get the following:

$$
\begin{aligned}
& \nabla_{\xi} \xi=0, \nabla_{\xi} e_{2}=0, \nabla_{\xi} e_{3}=0, \nabla_{\xi} e_{4}=0, \nabla_{\xi} e_{5}=\xi, \\
& \nabla_{e_{2}} \xi=2 e_{2}, \nabla_{e_{2}} e_{2}=-2 \xi, \nabla_{e_{2}} e_{3}=0, \nabla_{e_{2}} e_{4}=0, \nabla_{e_{2}} e_{5}=0, \\
& \nabla_{e_{3}} \xi=2 e_{3}, \nabla_{e_{3}} e_{2}=0, \nabla_{e_{3}} e_{3}=-2 \xi, \nabla_{e_{3}} e_{4}=0, \nabla_{e_{3}} e_{5}=0, \\
& \nabla_{e_{4}} \xi=0, \nabla_{e_{4}} e_{2}=0, \nabla_{e_{4}} e_{3}=0, \nabla_{e_{4}} e_{4}=0, \nabla_{e_{4}} e_{5}=0, \\
& \nabla_{e_{5}} \xi=0, \nabla_{e_{5}} e_{2}=0, \nabla_{e_{5}} e_{3}=0, \nabla_{e_{5}} e_{4}=0, \nabla_{e_{5}} e_{5}=0 .
\end{aligned}
$$

In view of the above relations we have

$$
\nabla_{X} \xi=-\phi^{2} X+h^{\prime} X
$$

for any $X \in \chi(M)$. Therefore, the structure $(\phi, \xi, \eta, g)$ is an almost contact metric structure such that $d \eta=0$ and $d \Phi=2 \eta \wedge \Phi$, so that $M$ is an almost Kenmotsu manifold.

By the above results, we can easily obtain the components of the curvature tensor $R$ as follows:

$$
\begin{aligned}
& R\left(\xi, e_{2}\right) \xi=4 e_{2}, R\left(\xi, e_{2}\right) e_{2}=-4 \xi, R\left(\xi, e_{3}\right) \xi=4 e_{3}, R\left(\xi, e_{3}\right) e_{3}=-4 \xi \\
& R\left(\xi, e_{4}\right) \xi=R\left(\xi, e_{4}\right) e_{4}=R\left(\xi, e_{5}\right) \xi=R\left(\xi, e_{5}\right) e_{5}=0 \\
& R\left(e_{2}, e_{3}\right) e_{2}=4 e_{3}, R\left(e_{2}, e_{3}\right) e_{3}=-4 e_{2}, R\left(e_{2}, e_{4}\right) e_{2}=R\left(e_{2}, e_{4}\right) e_{4}=0, \\
& R\left(e_{2}, e_{5}\right) e_{2}=R\left(e_{2}, e_{5}\right) e_{5}=R\left(e_{3}, e_{4}\right) e_{3}=R\left(e_{3}, e_{4}\right) e_{4}=0 \\
& R\left(e_{3}, e_{5}\right) e_{3}=R\left(e_{3}, e_{5}\right) e_{5}=R\left(e_{4}, e_{5}\right) e_{4}=R\left(e_{4}, e_{5}\right) e_{5}=0
\end{aligned}
$$

With the help of the expressions of the curvature tensor we conclude that the characteristic vector field $\xi$ belongs to the $(k, \mu)^{\prime}$-nullity distribution, with $k=-2$ and $\mu=-2$.

\section{References}

[1] Blair, D.E., Contact manifold in Riemannian geometry. Lecture Notes on Mathematics. Springer, Berlin, $509,1976$.

[2] Blair, D.E., Riemannian geometry on contact and symplectic manifolds. Progr. Math. 203, Birkhäuser, 2010.

[3] Blair, D.E., Koufogiorgos, T. and Papantoniou, B.J., Contact metric manifolds satisfying a nullity condition. Israel J. Math. 91 (1995), $189-214$.

[4] De, U.C., On $\phi$-symmetric Kenmotsu manifolds. Int. Electron. J. Geom. 1 (2008), 33-38.

[5] De, U.C., Sarkar, A., On $\phi$-Ricci symmetric Sasakian manifolds. Proc. of the Jangjeon Math. Soc. 11 (2008), 47-52. 
[6] De, U.C., Shaikh, A.A. and Biswas, S., On $\phi$-recurrent Sasakian manifolds. Novi Sad J. Math. 33 (2003), $43-48$.

[7] De, U.C., Yildiz, A. and Yaliniz, A.F., On $\phi$-recurrent Kenmotsu manifolds. Turkish J. Math. 33 (2009), 17-25.

[8] De, U.C., Yildiz, A. and Yaliniz, A.F., Locally $\phi$-symmetric normal almost contact metric manifolds of dimension 3. Applied Math. Letters. 22 (2009), 723-727.

[9] Dileo, G. and Pastore, A.M., Almost Kenmotsu manifolds and local symmetry. Bull. Belg. Math. Soc. Simon Stevin. 14 (2007), 343-354.

[10] Dileo, G. and Pastore, A.M., Almost Kenmotsu manifolds and nullity distributions. J. Geom. 93 (2009), 46-61.

[11] Dileo, G. and Pastore, A.M., Almost Kenmotsu manifolds with a condition of $\eta$-parallelism. Differential Geom. Appl. 27 (2009), 671-679.

[12] Gray, A., Spaces of constancy of curvature operators. Proc. Amer. Math. Soc. 17 (1966), 897-902.

[13] Kenmotsu, K., A class of almost contact Riemannian manifolds. Tohoku Math. J. 24 (1972), 93-103.

[14] Pastore, A.M. and Saltarelli, V., Generalized nullity distributions on almost Kenmotsu manifolds. Int. Electron. J. Geom. 4 (2011), $168-183$.

[15] Takahashi, T., Sasakian $\phi$-symmetic spaces. Tohoku Math. J. 29 (1977), 91-113.

[16] Tanno, S., Some differential equations on Riemannian manifolds. J. Math. Soc. Japan. 30 (1978), 509-531.

[17] Wang, Y. and Liu, X., Second order parallel tensors on almost Kenmotsu manifolds satisfying the nullity distributions. Filomat. 28 (2014), 839-847.

[18] Wang, Y. and Liu, X., Riemannian semisymmetric almost Kenmotsu manifolds and nullity distributions. Ann. Polon. Math. 112 (2014), 37-46.

[19] Wang, Y. and Liu, X., On $\phi$-recurrent almost Kenmotsu manifolds. Kuwait J. Sci. 42 (2015), 65-77.

[20] Wang, Y. and Liu, X., On a type of almost Kenmotsu manifolds with harmonic curvature tensors. Bull. Belg. Math. Soc. Simon Stevin. 22 (2015), 15-24.

[21] Wang, Y. and Liu, X., On almost Kenmotsu manifolds satisfying some nullity distributions. Proc. Natl. Acad. Sci., India, Sect. A Phys. Sci. 86 (2016), 347-353.

\section{Affiliations}

U.C. DE

AdDREss: Department of Pure Mathematics, University of Calcutta, 35, Ballygunge Circular Road, Kol700019, West Bengal, INDIA.

E-MAIL: uc_de@yahoo.com

KRISHANU MANDAL

AdDRESS: Department of Pure Mathematics, University of Calcutta, 35, Ballygunge Circular Road, Kol700019, West Bengal, INDIA.

E-MAIL: krishanu.mandal013@gmail.com 